\title{
Practical method to obtain a lower bound to the three-tangle
}

\author{
Christopher Eltschka ${ }^{1}$ and Jens Siewert ${ }^{2,3, *}$ \\ ${ }^{1}$ Institut für Theoretische Physik, Universität Regensburg, D-93040 Regensburg, Germany \\ ${ }^{2}$ Departamento de Química Física, Universidad del País Vasco UPVIEHU, E-48080 Bilbao, Spain \\ ${ }^{3}$ IKERBASQUE, Basque Foundation for Science, E-48011 Bilbao, Spain \\ (Received 30 October 2013; published 12 February 2014)
}

\begin{abstract}
The quantitative assessment of the entanglement in multipartite quantum states is, apart from its fundamental importance, a practical problem. Recently there has been significant progress in developing new methods to determine certain entanglement measures. In particular, there is a method-in principle, analytical - to compute a certified lower bound for the three-tangle. The purpose of this work is to provide a manual for the implementation of this approach and to explicitly discuss several analytically solvable cases in order to gauge the numerical tools. Moreover, we derive a simple analytical bound for the mixed-state three-tangle.
\end{abstract}

DOI: 10.1103/PhysRevA.89.022312

PACS number(s): 03.67.Mn, 03.65.Ud

\section{INTRODUCTION}

The quantitative theory of multipartite entanglement [1-3] is far from being a mature subject. The three-qubit problem, i.e., the quantification of Greenberger-Horne-Zeilinger (GHZ) entanglement in an arbitrary three-qubit state, may serve as an acid test for progress in this field. Recent years have brought substantial advancements regarding methods to estimate entanglement measures [4-17], on the one hand, and in terms of exact solutions of very specific problems [18-25], on the other hand. Although a mathematical insider of such methods would be able to produce lower bounds on the GHZtype entanglement in some generic cases the problem for the practitioner has remained open: Given an arbitrary three-qubit mixed state, e.g., the density matrix of an experimentally prepared state (such as in recent experiments [26-29]), determine the quality of the GHZ-type entanglement.

The objective of this article is to fill this gap. In a recent publication [30] we have proposed a method to quantify GHZtype entanglement in arbitrary $N$-qubit states. The three-qubit case was spelled out in some detail, however, not at the level at which one could directly apply it without some in-depth study of the background. In contrast, the present work focuses on the practical application of these findings. We explain the ingredients of the method and the steps one has to go through in order to implement the approach. Moreover, we provide an approximate analytical formula as well as a basic error estimate.

Why is it so important to obtain a lower bound? As the three-tangle for mixed states-like many other entanglement measures-is defined as a convex roof (see below), it is a minimum taken over all possible decompositions of a given state. Hence, any decomposition gives an upper bound, however, it is difficult to say by how much this bound overestimates the true three-tangle. In particular, knowing only upper bounds it cannot be excluded that the three-tangle vanishes. Therefore it is essential to obtain a lower bound. Once a nontrivial lower bound is known it becomes meaningful to look for good upper bounds as well.

\footnotetext{
*jens.siewert@ehu.es
}

The article is organized as follows: In Sec. II, we start by outlining the method and the most important concepts it requires. These concepts are then explained in separate parts: GHZ-symmetric states and the quantification of their three-tangle (Sec. III), symmetrization of an arbitrary state (Sec. IV), and analytical estimation of its three-tangle and entanglement optimization (Sec. V). In Sec. VI we highlight some experimental aspects of our method, and in Sec. VII we present an error estimate. Finally, in Sec. VIII we discuss the performance of our approach by applying it to several exactly solvable cases of three-qubit states or families.

\section{THE METHOD AND ITS INGREDIENTS}

In this section we introduce all the concepts and ideas that are required to solve our task: to estimate the amount of GHZtype entanglement in three-qubit states. While we just mention them here, they are considered and explained in detail in the following sections.

We exclusively consider three-qubit mixed states $\rho \in$ $\mathcal{B}(\mathcal{H})$, that is, positive definite Hermitian operators that act on the Hilbert space of three qubits, $\mathcal{H}=\mathbb{C}^{2} \otimes \mathbb{C}^{2} \otimes \mathbb{C}^{2}$. Quantification of the entanglement in $\rho$ makes sense only if the states are normalized: $\operatorname{tr} \rho=1$. Sometimes, however, we encounter states that are not normalized.

Suppose we are given a generic three-qubit state $\rho$ and would like to determine how much GHZ-type entanglement it contains. The appropriate entanglement measure for this purpose is the three-tangle [22,24,31,32], denoted $\tau_{3}$. It is the square root of the residual tangle originally introduced by Coffman et al. [31].

A lower bound to the exact $\tau_{3}(\rho)$ can be found by proceeding according to the following recipe:

(1) Find the so-called normal form of $\rho$. We denote the normal form $\rho^{\mathrm{NF}}$. As this operation in general is nonunitary we have $\operatorname{tr} \rho^{\mathrm{NF}} \leqq 1$. If $\operatorname{tr} \rho^{\mathrm{NF}}=0$, the procedure terminates here and $\tau_{3}(\rho)=0$.

(2) Optimize $\rho^{\mathrm{NF}} / \operatorname{tr} \rho^{\mathrm{NF}}$ by applying local unitary operations to the qubits according to an appropriate criterion (see below). The result is $\tilde{\rho}^{\mathrm{NF}}$.

(3) Project $\tilde{\rho}^{\mathrm{NF}}$ onto the GHZ-symmetric states and read off the value for $\tau_{3}$. The result $\tau_{3}\left(\mathbf{P}\left(\tilde{\rho}^{\mathrm{NF}}\right)\right)$ leads to the desired 
lower bound:

$$
\tau_{3}\left(\mathbf{P}\left(\tilde{\rho}^{\mathrm{NF}}\right)\right) \operatorname{tr} \rho^{\mathrm{NF}} \leqq \tau_{3}(\rho) .
$$

The idea behind this sequence is simple. In step 3, the state is projected onto a family of symmetric states for which the exact three-tangle is known. In this projection, generally entanglement is lost, but never gained. In order to minimize the entanglement loss one has to optimize the state-which is the purpose of steps 1 and 2 .

\section{THREE-TANGLE OF GHZ-SYMMETRIC STATES}

The GHZ-symmetric states [33], denoted $\rho^{\mathrm{S}}$, constitute the aforementioned peculiar family of symmetric states that facilitates the entire method described in this article. This is because the elements of this family contain much of the interesting physics of three-qubit states, but at the same time they are mathematically simple enough that many results can be calculated analytically.

\section{A. GHZ-symmetric three-qubit states}

The GHZ symmetry comprises the operations under which the well-known GHZ state

$$
|\mathrm{GHZ}\rangle=\frac{1}{\sqrt{2}}(|000\rangle+|111\rangle)
$$

remains invariant, that is,

(i) qubit permutations,

(ii) simultaneous three-qubit flips (i.e., application of $\sigma_{x} \otimes$ $\left.\sigma_{x} \otimes \sigma_{x}\right)$, and

(iii) qubit rotations about the $z$ axis of the form

$$
U\left(\phi_{1}, \phi_{2}\right)=\mathrm{e}^{\mathrm{i} \phi_{1} \sigma_{z}} \otimes \mathrm{e}^{\mathrm{i} \phi_{2} \sigma_{z}} \otimes \mathrm{e}^{-\mathrm{i}\left(\phi_{1}+\phi_{2}\right) \sigma_{z}} .
$$

Here, $\sigma_{x}$ and $\sigma_{z}$ are Pauli matrices. The only pure states invariant under this symmetry are

$$
\left|\mathrm{GHZ}_{ \pm}\right\rangle \equiv \frac{1}{\sqrt{2}}(|000\rangle \pm|111\rangle),
$$

while all other GHZ-symmetric states are mixed and, in the computational basis, are $8 \times 8$ matrices of the shape

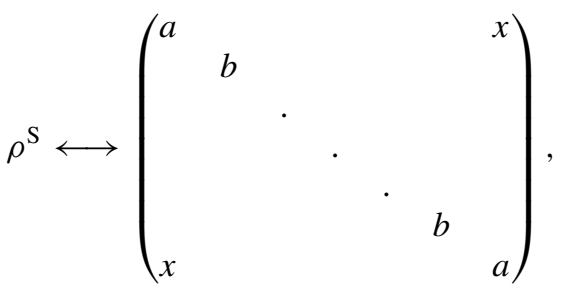

with $a, b, x \in \mathbb{R}$. As $\operatorname{tr} \rho^{\mathrm{S}}=1$ there are effectively two real parameters that characterize the entire family. A particularly nice (though not obvious) choice of parameters is

$$
\begin{aligned}
x\left(\rho^{\mathrm{S}}\right) & =\frac{1}{2}\left[\left\langle\mathrm{GHZ}_{+}\left|\rho^{\mathrm{S}}\right| \mathrm{GHZ}_{+}\right\rangle-\left\langle\mathrm{GHZ}_{-}\left|\rho^{\mathrm{S}}\right| \mathrm{GHZ}_{-}\right\rangle\right] \\
y\left(\rho^{\mathrm{S}}\right) & =\frac{1}{\sqrt{3}}\left[\left\langle\mathrm{GHZ}_{+}\left|\rho^{\mathrm{S}}\right| \mathrm{GHZ}_{+}\right\rangle+\left\langle\mathrm{GHZ}_{-}\left|\rho^{\mathrm{S}}\right| \mathrm{GHZ}_{-}\right\rangle-\frac{1}{4}\right]
\end{aligned}
$$



FIG. 1. (Color online) The family of GHZ-symmetric states $\rho^{\mathrm{S}}$. In the upper corners are the (pure) states $\mathrm{GHZ}_{ \pm}$, whereas the lower corner represents the separable mixture $\rho_{001}^{\mathrm{S}}=$ $\frac{1}{6} \sum_{j k l=001}^{110}|j k l\rangle\langle j k l|$. The GHZ-type states (GHZ) are separated from the states of at most $W$ type $(W)$ by the GHZ- $W$ line (5) [solid (red) line]. Note the symmetry $x \longleftrightarrow-x$ of the entanglementrelated properties. For a GHZ-type state $\rho^{\mathrm{S}}=\rho^{\mathrm{S}}\left(x_{0}, y_{0}\right)$ we indicate the optimal decomposition for the three-tangle [cf. Eq. (8)] consisting of state $\mathrm{GHZ}_{+}$and the mixed $W$-type state $\rho^{\mathrm{S}}\left(x_{0}^{W}, y_{0}^{W}\right)$.

such that the geometry in the space of density matrices induced by the Hilbert-Schmidt metric

$$
d_{\mathrm{HS}}^{2}(A, B) \equiv \frac{1}{2} \operatorname{tr}(A-B)^{\dagger}(A-B)
$$

coincides with the Euclidean geometry of the $x y$ plane in Fig. 1. For the parameters in Eq. (2) we have $a=\frac{1}{8}+\frac{\sqrt{3} y}{2}$ and $b=\frac{1}{8}-\frac{y}{2 \sqrt{3}}$.

As is well known there are two classes of three-qubit entangled states, the GHZ class and the $W$ class [34,35]. In Ref. [33] it was shown that the GHZ-W line, i.e., the border between these two classes, can be calculated exactly for GHZ-symmetric states (cf. Fig. 1). The corresponding parametrized curve is

$$
x^{W}=\frac{v^{5}+8 v^{3}}{8\left(4-v^{2}\right)}, \quad y^{W}=\frac{\sqrt{3}}{4} \frac{4-v^{2}-v^{4}}{4-v^{2}},
$$

with $-1 \leqq v \leqq 1$.

\section{B. Exact three-tangle}

Coffman et al. discovered an entanglement measure for three qubits that distinguishes between GHZ-type and $W$-type states, the residual tangle [31]. As mentioned before, taking the square root of this quantity has many advantages, and we refer to it as the three-tangle. For pure states it is defined as

$$
\begin{aligned}
\tau_{3}(\psi)= & 2 \sqrt{\left|d_{1}-2 d_{2}+4 d_{3}\right|} \\
d_{1}= & \psi_{000}^{2} \psi_{111}^{2}+\psi_{001}^{2} \psi_{110}^{2}+\psi_{010}^{2} \psi_{101}^{2}+\psi_{011}^{2} \psi_{100}^{2} \\
d_{2}= & \psi_{000} \psi_{001} \psi_{110} \psi_{111}+\psi_{000} \psi_{010} \psi_{101} \psi_{111} \\
& +\psi_{000} \psi_{011} \psi_{100} \psi_{111}+\psi_{001} \psi_{010} \psi_{101} \psi_{110} \\
& +\psi_{001} \psi_{011} \psi_{100} \psi_{110}+\psi_{010} \psi_{011} \psi_{100} \psi_{101} \\
d_{3}= & \psi_{000} \psi_{110} \psi_{101} \psi_{011}+\psi_{100} \psi_{010} \psi_{001} \psi_{111}
\end{aligned}
$$

Here $\psi_{j k l}$ with $j, k, l \in\{0,1\}$ are the components of a pure three-qubit state in the computational basis. It is easily checked 


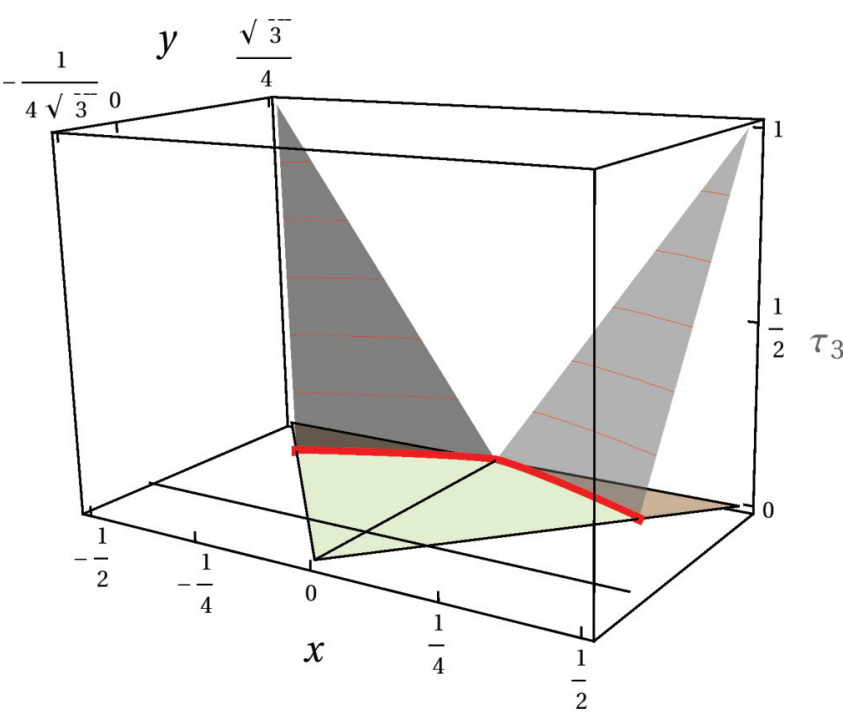

FIG. 2. (Color online) The exact three-tangle for the family of GHZ-symmetric states $\rho^{\text {S }}$; cf. Eq. (8).

that

$$
\tau_{3}(\mathrm{GHZ})=1 \quad \text { and } \quad \tau_{3}(W)=0,
$$

where $|W\rangle=\frac{1}{\sqrt{3}}(|001\rangle+|010\rangle+|100\rangle)$.

The three-tangle for mixed states is more complicated, as it is defined as a convex roof [36]

$$
\tau_{3}(\rho)=\min _{\text {all decomp. }} \sum p_{j} \tau_{3}\left(\psi_{j}\right)
$$

i.e., the minimum average three-tangle taken over all possible pure-state decompositions $\left\{p_{j}, \psi_{j}\right\}$ for $\rho=\sum_{j} p_{j}\left|\psi_{j}\right\rangle\left\langle\psi_{j}\right|$. This is what makes the computation of the three-tangle for mixed states difficult. For GHZ-symmetric three-qubit states, however, the convex roof of the three-tangle can be found exactly as [25]

$$
\begin{aligned}
& \tau_{3}\left(x_{0}, y_{0}\right) \\
& \quad= \begin{cases}0 & \text { for } x_{0}<x_{0}^{W} \text { and } y_{0}<y_{0}^{W}, \\
\frac{x_{0}-x_{0}^{W}}{\frac{1}{2}-x_{0}^{W}}=\frac{y_{0}-y_{0}^{W}}{\frac{\sqrt{3}}{4}-y_{0}^{W}} & \text { otherwise. }\end{cases}
\end{aligned}
$$

Here $x_{0} \geqq 0$ and $\left(x_{0}^{W}, y_{0}^{W}\right)$ are the coordinates of the intersection of the GHZ- $W$ line with the direction that contains both $\mathrm{GHZ}_{+}$and $\rho^{\mathrm{S}}\left(x_{0}, y_{0}\right)$ (cf. Fig. 1). The surface in Fig. 2 arises by connecting each point of the GHZ-W line $\left(x^{W}, y^{W}, \tau_{3}=\right.$ $0)$ with the closest of the points $\left(x_{\mathrm{GHZ}_{ \pm}}= \pm \frac{1}{2}, y_{\mathrm{GHZ}_{ \pm}}=\right.$ $\left.\frac{\sqrt{3}}{4}, \tau_{3}=1\right)$. That is, it interpolates linearly between the points of the GHZ-W line and the maximally entangled states $\mathrm{GHZ}_{ \pm}$.

\section{An analytical approximation}

We note that the nonvanishing curvature of the GHZ-W line, Eq. (5), is at the origin of the difficulty in writing down a more explicit formula than Eq. (8) for $\tau_{3}\left(\rho^{\mathrm{S}}\right)$. However, it is possible to find analytical approximations.

In analogy with the discussion in Ref. [30] we find a plane that approximates the surface in Eq. (8). The evident choice is a plane that contains the point $\left(x=\frac{1}{2}, y=\frac{\sqrt{3}}{4}, \tau_{3}=1\right)$ and

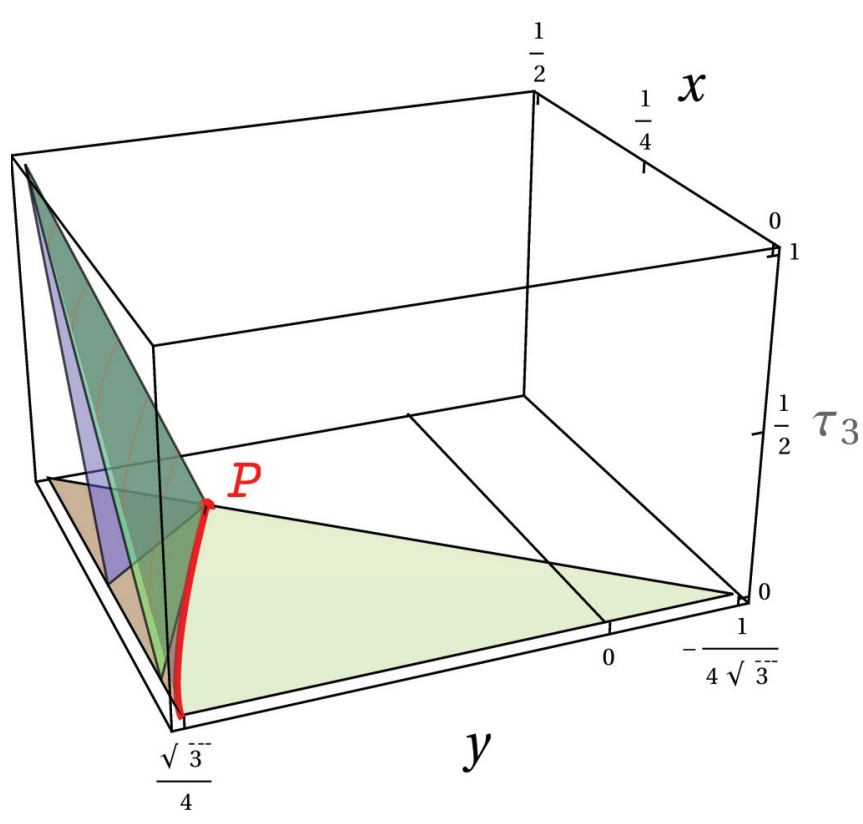

FIG. 3. (Color online) Simple quantitative witnesses for the three-tangle of GHZ-symmetric states $\rho^{\mathrm{S}}$. GHZ $\mathrm{GH}_{+}$is located in the upper-left corner of the $x y$ plane. Point $P$ has the coordinates $(x=$ $\frac{3}{8}, y=\frac{\sqrt{3}}{6}$ ). The uppermost tilted plane (blue) represents the quantitative witness $\tau_{3}=-4 \operatorname{tr}\left(\mathcal{W} \rho^{\mathrm{S}}\right)$, where $\mathcal{W}=\frac{3}{4} \mathbb{1}_{8}-|\mathrm{GHZ}\rangle\langle\mathrm{GHZ}|$ is the well-known projector-based GHZ witness [35]. An obviously better witness is $\mathcal{W}_{+}$, Eq. (9). The corresponding plane (green), Eq. (10), contains the tangent to the GHZ- $W$ line in point $P$ and is rather close to the exact solution, Eq. (8) (dark-green surface in the foreground).

that intersects the $x y$ plane in a straight line tangential to the GHZ- $W$ line. Each such tangent is an optimal GHZ witness [37]. Particularly simple tangents are given by the witnesses ( \pm for $x \gtrless 0$ )

$$
\mathcal{W}_{ \pm}=\frac{3}{4} \mathbb{1}-\left|\mathrm{GHZ}_{ \pm}\right\rangle\left\langle\mathrm{GHZ}_{ \pm}\left|-\frac{3}{7}\right| \mathrm{GHZ}_{\mp}\right\rangle\left\langle\mathrm{GHZ}_{\mp}\right|,
$$

which describe via $\operatorname{tr}\left(\rho^{\mathrm{S}} \mathcal{W}_{ \pm}\right)=0$ the tangents touching the GHZ- $W$ line in points $\left(x= \pm \frac{3}{8}, y=\frac{\sqrt{3}}{6}\right)$, respectively. The planes that contain one such tangent and the corresponding three-tangle point for $\mathrm{GHZ}_{ \pm}$are easily found (see Fig. 3) and give

$$
\tau_{3}^{\text {approx }}(x, y)=\max \left(0, \frac{4}{7}[-4+4|x|+5 \sqrt{3} y]\right)
$$

as our analytical approximation to Eq. (8) for the three-tangle of GHZ-symmetric states. It is exact for all states that lie on the lower edges of the triangle. In principle, each optimal witness of the type (9) from Ref. [37] gives rise to an approximation analogous to Eq. (10). They differ in the lines (in the $x y$ plane) for which they become exact.

\section{SYMMETRIZING AN ARBITRARY STATE}

Up to this point our discussion has been restricted to states $\rho^{\mathrm{S}}$ with symmetries i-iii (Sec. III A). A common way to extend our methods to arbitrary (nonsymmetric) states $\rho$ is by applying the projection $\mathbf{P}: \rho \mapsto \rho^{\mathrm{S}}$ onto the symmetric 
states. The operation

$$
\mathbf{P}(\rho)=\int \mathrm{d} U_{\mathrm{GHZ}} U_{\mathrm{GHZ}} \rho U_{\mathrm{GHZ}}^{\dagger}
$$

is often referred to as a twirling operation [38]. It averages over all symmetry elements, $\mathrm{i}-\mathrm{iii}$.

The effect of the projection $\mathbf{P}$ is easy to see. The matrix elements of the image of $\rho$ are

$$
\begin{aligned}
& \rho_{000,000}^{\mathrm{S}}=\rho_{111,111}^{\mathrm{S}}=\frac{1}{2}\left(\rho_{000,000}+\rho_{111,111}\right), \\
& \rho_{j k l, j k l}^{\mathrm{S}}=\frac{1}{6} \sum_{j k l=001}^{110} \rho_{j k l, j k l}, \\
& \rho_{000,111}^{\mathrm{S}}=\rho_{111,000}^{\mathrm{S}}=\frac{1}{2}\left(\rho_{000,111}+\rho_{111,000}\right), \\
& \rho_{j k l, m n r}^{\mathrm{S}}=0 \quad \text { for all other matrix elements. }
\end{aligned}
$$

From this the coordinates of the projection image in the $x y$ plane in Fig. 1 are readily obtained [33]:

$$
\begin{gathered}
x(\rho)=\frac{1}{2}\left(\rho_{000,111}+\rho_{111,000}\right), \\
y(\rho)=\frac{1}{\sqrt{3}}\left(\rho_{000,000}+\rho_{111,111}-\frac{1}{4}\right) .
\end{gathered}
$$

It is worth noting that these relations, inserted into Eq (10), give a simple explicit formula to approximate the three-tangle of an arbitrary mixed state:

$$
\begin{aligned}
\tau_{3}^{\text {approx }}(\rho)= & \max \left(0,\left[ \pm \frac{8}{7}\left(\rho_{000,111}+\rho_{111,000}\right)\right.\right. \\
& \left.\left.+\frac{20}{7}\left(\rho_{000,000}+\rho_{111,111}\right)-3\right]\right)
\end{aligned}
$$

Clearly, the performance of this formula is better the closer state $\rho$ is to a GHZ-symmetric state. However, the local bases will not always be arranged this way. Therefore, the result for $\tau_{3}^{\text {approx }}$ can be improved by applying local unitaries to $\rho$.

We have already mentioned that symmetrizing a state usually results in a loss of three-tangle. In order to see this consider the GHZ state $\frac{1}{\sqrt{2}}(|100\rangle+|011\rangle)$. Symmetrization maps it to the lower corner of the triangle in Fig. 1, i.e., to a completely separable state. Naturally we would like to avoid such entanglement losses. This is why we have to optimize the state before projecting it. While optimization cannot exclude entanglement loss, it may reduce it substantially. In the example, the optimization is particularly simple. Applying $\sigma_{x}$ (a local operation that does not change entanglement) to the first qubit yields $\mathrm{GHZ}_{+}$, which preserves its full entanglement under projection $\mathbf{P}$.

\section{OPTIMIZING THE STATE}

The example in the preceding section shows that projection onto the GHZ-symmetric states may serve to quantitatively assess the three-tangle of a state and that optimization prior to projection may enhance the reliability of the result. Clearly, symmetrization can only reduce, and never enhance, the entanglement of a state [33]. Therefore, if the projection image $\rho^{\mathrm{S}}$ contains a three-tangle, then the original state $\rho$ also does. This is why we also call this method a quantitative witness.
The idea that witnessing entanglement can be improved by optimization was discussed by various authors, e.g., in Refs. [6,7,39].

At first glance it looks difficult to find generally valid criteria to improve an arbitrary state. In this section, we sketch why optimization steps 1 and 2 are appropriate for achieving this goal.

\section{A. Normal form}

The normal form of a multipartite quantum state is a fundamental concept that was introduced by Verstraete et al. [32] and discussed in detail also in Ref. [40]. It applies to arbitrary (finite-dimensional) multiqudit states. Here we focus on $N$-qubit states only. The essential merit of the normal form in the present context is that, among all states that are locally equivalent to the original state, it is the one that maximizes a certain type of entanglement measures such as the three-tangle. Let us elaborate on this point.

The defining property of the normal form $\rho^{\mathrm{NF}}$ is that all local density matrices are proportional to the identity

$$
\left(\rho^{\mathrm{NF}}\right)_{(j)}=\operatorname{tr}_{1 \ldots(j-1)(j+1) \ldots N} \rho^{\mathrm{NF}} \propto \mathbb{1}_{2} .
$$

Therefore the normal form is unique only up to local unitaries. Local equivalence to the original state $\rho$ means that $\rho^{\mathrm{NF}}$ can be obtained from $\rho$ by applying local operations,

$$
\rho^{\mathrm{NF}}=\left(A_{1} \otimes \ldots \otimes A_{N}\right) \rho\left(A_{1} \otimes \ldots \otimes A_{N}\right)^{\dagger} .
$$

Here the $A_{j}$ are invertible single-qubit operations that are not necessarily norm preserving. For the normal form we additionally require $\operatorname{det} A_{j}=1$ or, more technically, $A_{j} \in \mathrm{SL}(2, \mathbb{C})$. Note that sometimes the normal form can be reached only asymptotically. This is especially true for all pure states with a vanishing three-tangle, where the normal form is simply 0 .

How can we practically find the normal form? There is a simple iterative procedure described in Ref. [32]. Let us denote the reduced local density matrix of the $j$ th qubit $\rho_{(j)}$. One starts by transforming $\rho$ according to

$$
\rho \rightarrow \frac{1}{\operatorname{det} \rho_{(1)}^{-1 / 2}}\left(\rho_{(1)}^{-1 / 2} \otimes \mathbb{1}_{2} \otimes \ldots\right) \rho\left(\rho_{(1)}^{-1 / 2} \otimes \mathbb{1}_{2} \otimes \ldots\right)^{\dagger},
$$

which brings $\rho_{(1)}$ into a form $\propto \mathbb{1}_{2}$. Next one applies the analogous step to the second qubit. Note that, while making the second qubit $\propto \mathbb{1}_{2}$ this property on the first qubit is usually destroyed. Then one continues with the third qubit, and so on, to the $N$ th qubit, and then all over again. The convergence of this procedure was proved in Ref. [32], and the result is the normal form. In each step of the iteration the trace is reduced (or unchanged). As the three-tangle remains unchanged under a transformation $A_{j}$ this means that, after renormalization of the transformed state, the three-tangle has increased. This is the reason why the normal form is a useful first optimization step for our purposes. If we normalize the normal form the resulting state $\rho^{\mathrm{NF}} / \operatorname{tr} \rho^{\mathrm{NF}}$ has maximal three-tangle in the local orbit of $\rho$ [32].

It is worth noting that GHZ-symmetric states-which play a central role in our discussion-are naturally given in their normal form. 


\section{B. Criteria for unitary optimization}

As the normal form is unique only up to local unitaries, another optimization is required in order to find the appropriate local qubit bases. That is, we transform the normalized state $\rho^{\mathrm{NF}} / \operatorname{tr} \rho^{\mathrm{NF}}$ by applying a unitary operation, $V \in \mathrm{SU}(2)^{\otimes 3}$ :

$$
\tilde{\rho}^{\mathrm{NF}}=V \frac{\rho^{\mathrm{NF}}}{\operatorname{tr} \rho^{\mathrm{NF}}} V^{\dagger} .
$$

The obvious criterion is to maximize the three-tangle,

$$
\tau_{3}\left(\mathbf{P}\left(\tilde{\rho}^{\mathrm{NF}}\right)\right) \longrightarrow \max ,
$$

that is,

$$
\tilde{\rho}^{\mathrm{NF}}=\underset{V \in \operatorname{SU}(2)^{\otimes 3}}{\operatorname{argmax}} \tau_{3}\left(\mathbf{P}\left(V \frac{\rho^{\mathrm{NF}}}{\operatorname{tr} \rho^{\mathrm{NF}}} V^{\dagger}\right)\right) .
$$

Clearly Eq. (15) gives the largest possible value of $\tau_{3}$. However, due to the complicated structure of the function in Eq. (8) it appears hopeless to obtain analytical results.

Alternatively one may choose optimization criteria that do not necessarily give the maximal value of $\tau_{3}$ but, depending on the state $\rho$, can possibly be treated analytically. Examples are the overlap (fidelity) with the GHZ state,

$$
\tilde{\rho}_{(\mathrm{f})}^{\mathrm{NF}}=\underset{V \in \mathrm{SU}(2)^{\otimes 3}}{\operatorname{argmax}}\left\langle\mathrm{GHZ}\left|V \frac{\rho^{\mathrm{NF}}}{\operatorname{tr} \rho^{\mathrm{NF}}} V^{\dagger}\right| \mathrm{GHZ}\right\rangle,
$$

and the Hilbert-Schmidt distance from the GHZ state,

$$
\tilde{\rho}_{(\mathrm{d})}^{\mathrm{NF}}=\underset{V \in \mathrm{SU}(2)^{\otimes 3}}{\operatorname{argmin}} d_{\mathrm{HS}}\left(\pi_{\mathrm{GHZ}}, V \frac{\rho^{\mathrm{NF}}}{\operatorname{tr} \rho^{\mathrm{NF}}} V^{\dagger}\right),
$$

where $\pi_{\mathrm{GHZ}} \equiv|\mathrm{GHZ}\rangle\langle\mathrm{GHZ}|$.

\section{EXPERIMENTAL ASPECTS}

In order to apply our method to an experimentally prepared state its density matrix needs to be determined. This is done using quantum-state tomography [41]. To this end we note that the density matrix can be represented in a basis of local Pauli operators,

$$
\rho=\frac{1}{8} \sum_{j k l} \hat{\rho}_{j k l} \sigma_{j} \otimes \sigma_{k} \otimes \sigma_{l},
$$

where $\hat{\rho}_{j k l}=\operatorname{tr}\left(\rho \sigma_{j} \otimes \sigma_{k} \otimes \sigma_{l}\right)$. Each term in Eq. (18) corresponds to a joint measurement of local observables. To obtain the optimized lower bound for the three-tangle (as described in the preceding section), full tomography is required. However, even in a small system like three qubits, full tomography requires the measurement of 63 observables ( + extra measurements for normalization) and is therefore expensive. There are ways to reduce the effort, as it is often the case that the experimentally prepared state is not completely unknown.

The first possibility is related to the observation that the analytical approximation, Eq. (14), contains only the four entries $\rho_{000,000}, \rho_{000,111}, \rho_{111,000}$, and $\rho_{111,111}$. In Ref. [42] the authors describe a general method to determine the fidelity with the GHZ state. For three qubits it requires four measurement settings. Those settings determine the diagonal elements and the real part of $\rho_{000,111}$ separately, so that they are indeed sufficient to calculate the projection of the state onto the GHZ symmetric states. If the off-diagonal matrix element is not known to be real, it is worthwhile determining the imaginary part as well and to use the absolute value instead of the real part (this is equivalent to a very restricted unitary optimization on the results). It turns out that the additional setting $Y Y Y$ (i.e., measurement of $\sigma_{y}$ for all three qubits) is sufficient for that purpose.

Note that the best choice of local bases is such that the fidelity of the GHZ state in those bases is maximized. In a sense, this corresponds to carrying out the local unitary optimization for Eq. (14) directly in the experiment, while it would not be possible numerically, as not all elements of the density matrix are known.

Another alternative to full tomography is permutationally invariant tomography (PIT) [43]. The experimental setup of PIT effectively means to apply the permutation averaging part of the twirling operation [described in Eq. (11)] directly in the experiment, before the measurement. Therefore using PIT will in general result in a worse lower bound. However, if the true state has approximate permutation symmetry-which will often be the case in experiments aiming at GHZ states-one may hope that only little entanglement information is lost in the projection $\rho \stackrel{\text { PIT }}{\longrightarrow} \rho^{(\mathrm{PI})}$. For three qubits 10 settings are required [43]. As the result of PIT is a valid density matrix the optimization procedure may be applied to $\rho^{(\mathrm{PI})}$ as well to improve the estimate of $\tau_{3}$.

Also, in the case of performing PIT it is possible to reduce the experimental effort by measuring only the four GHZ matrix elements. If we content ourselves with the real part of the measurement only, the three settings $Z Z Z$, $X X X$, and $X Y Y$ are sufficient. The real part of the offdiagonal element is determined from $\langle X X X\rangle_{\mathrm{PIT}}=\langle X X X\rangle$ and $\langle X Y Y\rangle_{\mathrm{PIT}}=\frac{1}{3}\langle X Y Y+Y X Y+Y Y X\rangle$ as $\operatorname{Re}\left(\rho_{000,111}\right)=$ $\langle X X X\rangle_{\mathrm{PIT}}-3\langle X Y Y\rangle_{\mathrm{PIT}}$. The imaginary part requires the additional settings $X X Y$ and $Y Y Y$, so that $\operatorname{Im}\left(\rho_{000,111}\right)=$ $\langle Y Y Y\rangle_{\mathrm{PIT}}-3\langle X X Y\rangle_{\mathrm{PIT}}$.

In summary, we see that there is a trade-off between the experimental effort and the quality of the lower bound for $\tau_{3}$. Obviously the best estimate is achieved by doing full tomography of the state and then applying the procedure in Sec. II to the result. In this case, the best possible lower bound with our method is achieved.

In the opposite extreme case, one measures the GHZ matrix elements $\rho_{000,000}, \rho_{111,111}, \operatorname{Re}\left(\rho_{000,111}\right)=\operatorname{Re}\left(\rho_{111,000}\right)$ with an experimental PIT setup using just three measurement settings in a specific local basis, however, at the cost that no further optimization of the bound is possible (but limited premeasurement optimization, by appropriately choosing the local measurement bases). Two additional measurement settings allow one also to determine $\operatorname{Im}\left(\rho_{000,111}\right)$, which may already improve the bound.

Between those two extreme possibilities there is full PIT (requiring much fewer settings than full tomography but maintaining the possibility of numerically optimizing the bound for $\tau_{3}$ ). We note that-since the three-setting and/or five-setting measurements use the same setup and a strict subset of the measurements for full PIT - they can be carried 
out first, and if this is not sufficient for a reasonable lower bound, one can proceed by doing full PIT without wasting experimental effort.

With standard tomography, the minimal effort is four measurement settings, which is slightly more than in a PIT setup. If the imaginary part of the off-diagonal matrix is desired as well, only one additional setting is required, so standard and PIT setups are equally efficient in that case.

\section{ESTIMATING THE ERROR OF THE LOWER BOUND}

As, in general, entanglement is lost in the projection $\mathbf{P}\left(\tilde{\rho}^{\mathrm{NF}}\right) \equiv \tilde{\rho}^{\mathrm{S}}$ it is desirable to get an idea by how much $\tau_{3}\left(\tilde{\rho}^{\mathrm{S}}\right)$ underestimates the three-tangle of $\tilde{\rho}^{\mathrm{NF}}$. Recall that we know the value $\tau_{3}\left(\tilde{\rho}^{\mathrm{S}}\right)$ exactly, and moreover, $\tilde{\rho}^{\mathrm{S}} \leqq \tilde{\rho}^{\mathrm{NF}}$. However, we do not know how much larger $\tau_{3}\left(\tilde{\rho}^{\mathrm{NF}}\right)$ actually is. Consequently we need an upper bound to $\tau_{3}\left(\tilde{\rho}^{\mathrm{NF}}\right)$ to estimate the error.

In principle, any available decomposition of $\tilde{\rho}^{\mathrm{NF}}$ provides an upper bound to $\tau_{3}\left(\tilde{\rho}^{\mathrm{NF}}\right)$. The simplest upper bound is $\tau_{3}\left(\tilde{\rho}^{\mathrm{NF}}\right) \leqq\left\langle\mathrm{GHZ}\left|\tilde{\rho}_{(\mathrm{f})}^{\mathrm{NF}}\right| \mathrm{GHZ}\right\rangle$. A good upper bound can be found numerically using elaborate methods as described in Refs. [9,11,14,17]. Alternatively, one can refine the simple estimate by applying a geometrical method that represents a variant of the ideas in Ref. [17]. It uses the convexity of both the state space and the three-tangle and is most easily explained by considering Fig. 4.

In the figure, the GHZ-symmetric states are represented by a line. They form a subset of the complete state space. The projection of the optimized state $\tilde{\rho}^{\mathrm{NF}}$ onto the GHZ-symmetric states is then just an orthogonal projection onto that line. We may assume $\tilde{\rho}^{\mathrm{NF}} \neq \tilde{\rho}^{\mathrm{S}}$; otherwise, we do not need an error estimate.

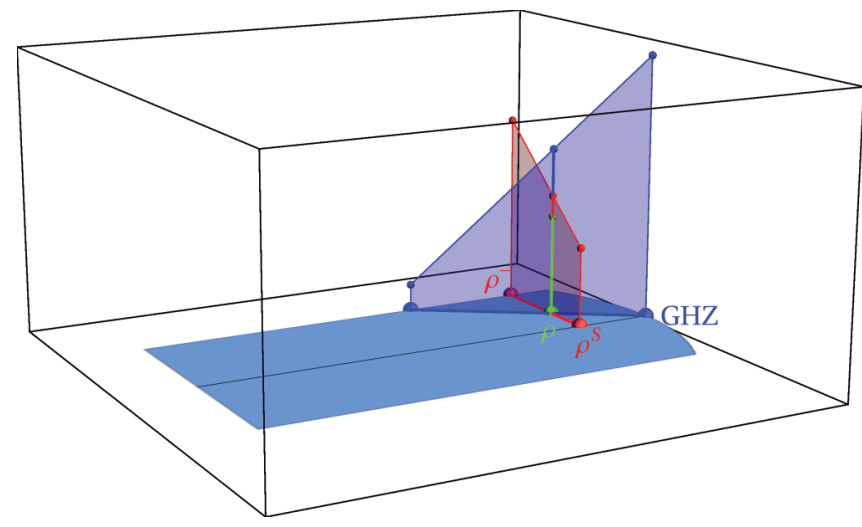

FIG. 4. (Color online) Illustration of the error estimate. The base area (blue) represents the set of all three-qubit states. The solid black line in it corresponds to the GHZ-symmetric states and has the state $\mathrm{GHZ}_{+}$at its right end point. A density matrix $\rho$ (light-green line) is projected by the twirling operation, Eq. (11), onto $\rho^{\mathrm{S}}$ (red line) in the GHZ-symmetric states, thereby reducing the three-tangle (which is displayed in the vertical direction). State $\rho_{-}$is found by extending the straight line from $\rho^{\mathrm{S}}$ through $\rho$ towards the border of the state space. An upper bound $\tau_{3}^{-} \geqq \tau_{3}\left(\rho_{-}\right)$yields an upper bound for $\tau_{3}(\rho)$ due to the convexity of the three-tangle. Alternatively, one may use any other state instead of $\rho^{\mathrm{S}}$ with a known three-tangle (for example, the GHZ state, as indicated by the other vertical plane).
Consider now the straight line connecting $\tilde{\rho}^{\mathrm{S}}$ and $\tilde{\rho}^{\mathrm{NF}}$. We extend this line until it reaches the border of the space of density matrices in state $\rho_{-}$. This means that $\rho_{-}$is an affine combination of $\tilde{\rho}^{\mathrm{S}}$ and $\tilde{\rho}^{\mathrm{NF}}$,

$$
\rho_{-}=\frac{1}{\lambda} \tilde{\rho}^{\mathrm{NF}}-\frac{1-\lambda}{\lambda} \tilde{\rho}^{\mathrm{S}},
$$

with some real $\lambda \in(0,1]$ that is defined by the condition that the smallest eigenvalue of $\rho_{-}$be 0 .

Given $\rho_{-}$we can determine an upper bound $\tau_{3}^{-} \geqq \tau_{3}\left(\rho_{-}\right)$ and obtain

$$
\tau_{3}\left(\tilde{\rho}^{\mathrm{NF}}\right) \leqq \lambda \tau_{3}^{-}+(1-\lambda) \tau_{3}\left(\tilde{\rho}^{\mathrm{S}}\right) .
$$

Evidently this method of estimating $\tau_{3}\left(\tilde{\rho}^{\mathrm{NF}}\right)$ can be applied analogously with any other state $\rho$ (in place of $\tilde{\rho}^{\mathrm{S}}$ ) for which the exact three-tangle is known, for example, the states $\mathrm{GHZ}_{ \pm}$.

\section{EXAMPLES}

In this section we illustrate and analyze the performance of our method by applying it to states whose three-tangle is known exactly or with a high numerical precision. Among other details, this reveals that our method gives exact results not just for GHZ-symmetric states.

\section{A. Mixtures of a GHZ state with a product state}

Consider mixtures of the GHZ state and an orthogonal product state

$$
\rho_{[1]}(p)=p|\mathrm{GHZ}\rangle\langle\mathrm{GHZ}|+(1-p)| 001\rangle\langle 001|,
$$

which we mentioned briefly in the Supplement to Ref. [30]. The exact solution $\tau_{3}\left(\rho_{[1]}(p)\right)=p$ can be derived following Refs. [19,24]. Here we give the analytical solution by means of the method discussed in the present article.

By applying the local operation

$$
A_{[1]}=\left(\begin{array}{cc}
\alpha & 0 \\
0 & 1 / \alpha
\end{array}\right) \otimes \mathbb{1}_{2} \otimes\left(\begin{array}{cc}
1 / \alpha & 0 \\
0 & \alpha
\end{array}\right)
$$

to $\rho_{[1]}$, we obtain

$$
A_{[1]} \rho_{[1]} A_{[1]}^{\dagger}=p|\mathrm{GHZ}\rangle\left\langle\mathrm{GHZ}\left|+(1-p) \alpha^{4}\right| 001\right\rangle\langle 001|,
$$

and letting $\alpha \rightarrow 0$ the normal form

$$
\rho_{[1]}^{\mathrm{NF}}(p)=p|\mathrm{GHZ}\rangle\langle\mathrm{GHZ}| .
$$

No further unitary optimization is required and the result is

$$
\tau_{3}\left(\rho_{[1]}(p)\right)=\operatorname{tr} \rho_{[1]}^{\mathrm{NF}}(p) \tau_{3}(\mathrm{GHZ})=p,
$$

showing that there are also cases of states for which the exact three-tangle is obtained, although they are not GHZ symmetric. This is not a coincidence. The states $\rho_{[1]}$ can be obtained from a two-qubit state simply by doubling the first qubit in each basis state (that is, $|j k\rangle\langle l m|\rightarrow| j j k\rangle\langle l l m|$ ). Multipartite entanglement in such states was analyzed in Ref. [44]. Consequently, these states belong to a subspace that can be described by two-qubit physics. However, as the method is exact for arbitrary two-qubit states [30], it must be exact also for $\rho_{[1]}$. 


\section{B. Mixtures of a GHZ state and a $W$ state}

Our second example is

$$
\rho_{[2]}(p)=p|\mathrm{GHZ}\rangle\langle\mathrm{GHZ}|+(1-p)| W\rangle\langle W|,
$$

for which the exact solution was found in Ref. [18] (for the residual tangle) and in Ref. [24] (for the three-tangle). The states contain a three-tangle for $p \geqq p_{0}=\frac{2^{1 / 3}}{2^{1 / 3}+3 / 4} \approx 0.627$ so that

$$
\tau_{3}\left(\rho_{[2]}(p)\right)=\max \left(0, \frac{p-p_{0}}{1-p_{0}}\right) .
$$

First, we note that $\rho_{[2]}$ is not in normal form, however, for $p \geqq p_{0}$ it is rather close to it. The local transformation to get the normal form is diagonal and can, in principle, be obtained analytically. To render the discussion more transparent we use the approximation

$$
\rho_{[2]}^{\mathrm{NF}}(p) \approx \rho_{[2]}(p) .
$$

Then, again, no unitary optimization is necessary and the symmetrized state is approximately

$$
\begin{aligned}
\tilde{\rho}_{[2]}^{\mathrm{S}}(p) \approx & p \pi_{\mathrm{GHZ}} \\
& +\frac{1-p}{6}\left(\pi_{001}+\pi_{010}+\pi_{100}+\pi_{011}+\pi_{101}+\pi_{110}\right),
\end{aligned}
$$

where $\pi_{k l m} \equiv|k l m\rangle\langle k l m|$. The states $\tilde{\rho}_{[2]}^{\mathrm{S}}(p)$ are located at the lower right border of the triangle in Fig. 1. They have a nonvanishing three-tangle for $p>3 / 4$, and hence,

$$
\tau_{3}\left(\tilde{\rho}_{[2]}(p)\right) \approx \max (0,4 p-3) .
$$

We clearly observe the loss of tripartite entanglement due to the projection; in the worst case (for $p=3 / 4$ ), it amounts to $\frac{3 / 4-p_{0}}{1-p_{0}} \approx 0.33$. Note also that the error estimate in Sec. VI is not helpful in a case like this: Since $\rho_{[2]}$ is of rank 2 it is located at the border of the state space for most directions. On the other hand, a considerable part of the large errors occurs for states $\rho_{[2]}$ that have a nonzero three-tangle, while for the estimate $\tau_{3}\left(\tilde{\rho}_{[2]}^{\mathrm{S}}\right)=0$. In such cases, a numerical method like that in Ref. [17] is highly useful, as it is capable of certifying (numerically) a vanishing three-tangle.

\section{A nontrivial $W$ state}

The last example we consider in this section is the state

$$
\rho_{[3]}=\frac{1}{8}\left(\begin{array}{llllllll}
1 & 0 & 0 & 0 & 0 & 0 & 0 & 0 \\
0 & 1 & 1 & 1 & 1 & 1 & 1 & 0 \\
0 & 1 & 1 & 1 & 1 & 1 & 1 & 0 \\
0 & 1 & 1 & 1 & 1 & 1 & 1 & 0 \\
0 & 1 & 1 & 1 & 1 & 1 & 1 & 0 \\
0 & 1 & 1 & 1 & 1 & 1 & 1 & 0 \\
0 & 1 & 1 & 1 & 1 & 1 & 1 & 0 \\
0 & 0 & 0 & 0 & 0 & 0 & 0 & 1
\end{array}\right) .
$$

It is a rank 3 state, a mixture of the GHZ-type state $|\varphi\rangle=$ $\frac{1}{\sqrt{6}}(|001\rangle+|010\rangle+\ldots+|110\rangle)$ and two product states, $\pi_{000}$ and $\pi_{111}$. It is difficult to decide at first glance which class $\rho_{[3]}$ belongs to.

Application of the procedure described in Sec. II yields, after projection to the GHZ-symmetric states, a point in the $W$ region. That is, the state is at least of $W$ type: The state $\tilde{\rho}_{[3]}^{\mathrm{NF}}$ before the projection is locally equivalent to $\rho_{[3]}$ and the image of the projection $\tilde{\rho}_{[3]}^{\mathrm{S}}$ has, at most, the class of the original state. However, we cannot be sure that its three-tangle indeed equals 0 .

We may try to find an explicit decomposition of $\rho_{[3]}$ that has a vanishing three-tangle. It turns out that a decomposition into three pure states of the form

$$
\left|\psi_{j}\right\rangle=c_{j 1}|000\rangle+c_{j 2}|\varphi\rangle+c_{j 3}|111\rangle
$$

is sufficient. A numerical minimization indeed gives a decomposition with a numerically vanishing three-tangle:

$$
\begin{aligned}
\rho_{[3]} \approx & 0.32809\left|\psi_{1}\right\rangle\left\langle\psi_{1}\right| \\
& +0.52694\left|\psi_{2}\right\rangle\left\langle\psi_{2}|+0.14498| \psi_{3}\right\rangle\left\langle\psi_{3}\right|,
\end{aligned}
$$

with

$$
\begin{aligned}
& \left|\psi_{1}\right\rangle \approx 0.45488|000\rangle-0.86186|\varphi\rangle-0.22423|111\rangle, \\
& \left|\psi_{2}\right\rangle \approx 0.28991|000\rangle+0.95673|\varphi\rangle-0.02476|111\rangle, \\
& \left|\psi_{3}\right\rangle \approx 0.29741|000\rangle-0.40662|\varphi\rangle+0.86383|111\rangle .
\end{aligned}
$$

(For a precision better than $\tau_{3}<10^{-20}$ many more digits of the coefficients need to be taken into account.)

Consequently, our method works well also for states whose optimal decompositions are more intricate. A possible reason for the reliability in the case of $\rho_{[3]}$ is the presence of both permutation and spin-flip invariance in the state.

\section{SUMMARY}

We have described the practical implementation of a method that yields a lower bound-in principle, analyticalto the three-tangle of an arbitrary three-qubit mixed state. While the theoretical grounds of this method were investigated in Refs. [30,33] we have focused here on various practical aspects. Apart from the ingredients of the method we have provided a more detailed discussion of the approximation to the three-tangle by projector-based entanglement witnesses (and the corresponding quantitative witnesses; Sec. III C), as well as an analytical lower bound for the three-tangle of an arbitrary three-qubit density matrix, Eq. (10). Moreover, we have considered the trade-off between the quality of the lower bound and the experimental effort to determine elements of the density matrix.

As the procedure explained here gives only a lower bound to the three-tangle, one would like to know about the possible error. To this end, we have described an error estimate based on simple geometrical considerations. Finally, we have studied the performance of the method by comparing its results to exactly solvable cases for the three-tangle. From this we may conclude that the method often works well; however, in particular, for a small three-tangle in the original state, the bound may substantially underestimate the entanglement. Therefore, it is useful to combine it with a numerical approach (such as in Ref. [17]) that can numerically certify a vanishing three-tangle.

\section{ACKNOWLEDGMENTS}

The authors thank P. J. Love for interesting discussions, C. Schwemmer and G. Tóth for helpful comments, and 
J. Fabian and K. Richter for their support. This work was funded by the German Research Foundation within SPP 1386
(C.E.), by Basque Government Grant No. IT-472-10, and by MINECO Grant No. FIS2012-36673-C03-01 (J.S.).
[1] M. B. Plenio and S. Virmani, Quant. Inf. Comput. 7, 1 (2007).

[2] L. Amico, R. Fazio, A. Osterloh, and V. Vedral, Rev. Mod. Phys. 80, 517 (2008).

[3] R. Horodecki, P. Horodecki, M. Horodecki, and K. Horodecki, Rev. Mod. Phys. 81, 865 (2009).

[4] R. Horodecki, M. Horodecki, and P. Horodecki, Phys. Rev. A 59, 1799 (1999).

[5] K. M. R. Audenaert and M. B. Plenio, New J. Phys. 8, 266 (2006).

[6] O. Gühne, M. Reimpell, and R. F. Werner, Phys. Rev. Lett. 98, 110502 (2007).

[7] J. Eisert, F. G. S. L. Brandão, and K. Audenaert, New J. Phys. 9, 46 (2007).

[8] A. Osterloh, J. Siewert, and A. Uhlmann, Phys. Rev. A 77, 032310 (2008).

[9] B. Röthlisberger, J. Lehmann, D. S. Saraga, P. Traber, and D. Loss, Phys. Rev. Lett. 100, 100502 (2008).

[10] A. Osterloh and P. Hyllus, Phys. Rev. A 81, 022307 (2010).

[11] K. Cao, Z.-W. Zhou, G.-C. Guo, and L. He, Phys. Rev. A 81, 034302 (2010).

[12] B. Jungnitsch, T. Moroder, and O. Gühne, Phys. Rev. Lett. 106, 190502 (2011).

[13] Z.-H. Ma, Z.-H. Chen, J.-L. Chen, C. Spengler, A. Gabriel, and M. Huber, Phys. Rev. A 83, 062325 (2011).

[14] J. Zhu, S. Kais, A. Aspuru-Guzik, S. Rodriques, B. Brock, and P. J. Love, J. Chem. Phys. 137, 074112 (2012).

[15] J.-Y. Wu, H. Kampermann, D. Bruss, C. Klöckl, and M. Huber, Phys. Rev. A 86, 022319 (2012).

[16] Z.-H. Chen, Z.-H. Ma, O. Gühne, and S. Severini, Phys. Rev. Lett. 109, 200503 (2012).

[17] S. Rodriques, N. Datta, and P. J. Love, arXiv:1307.2323.

[18] R. Lohmayer, A. Osterloh, J. Siewert, and A. Uhlmann, Phys. Rev. Lett. 97, 260502 (2006).

[19] C. Eltschka, A. Osterloh, J. Siewert, and A. Uhlmann, New J. Phys. 10, 043014 (2008).

[20] E. Jung, M. R. Hwang, D. Park, and J. W. Son, Phys. Rev. A 79, 024306 (2009).

[21] E. Jung, D. Park, and J. W. Son, Phys. Rev. A 80, 010301(R) (2009).

[22] G. Gour, Phys. Rev. Lett. 105, 190504 (2010).

[23] S. J. He, X. H. Wang, S. M. Fei, H. X. Sun, and Q. Y. Wen, Comm. Theoret. Phys. 55, 251 (2011).
[24] O. Viehmann, C. Eltschka, and J. Siewert, Appl. Phys. B 106, 533 (2012).

[25] J. Siewert and C. Eltschka, Phys. Rev. Lett. 108, 230502 (2012).

[26] M. Neeley, R. C. Bialczak, M. Lenander, E. Lucero, M. Mariantoni, A. D. O'Connell, D. Sank, H. Wang, M. Weides, J. Wenner, Y. Yin, T. Yamamoto, A. N. Cleland, and J. M. Martinis, Nature 467, 570 (2010).

[27] L. DiCarlo, M. D. Reed, L. Sun, B. R. Johnson, J. M. Chow, J. M. Gambetta, L. Frunzio, S. M. Girvin, M. H. Devoret, and R. J. Schoelkopf, Nature 467, 574 (2010).

[28] M. Baur, A. Fedorov, L. Steffen, S. Filipp, M. P. da Silva, and A. Wallraff, Phys. Rev. Lett. 108, 040502 (2012).

[29] P. Schindler, T. Monz, D. Nigg, Julio T. Barreiro, E. A. Martinez, M. F. Brandl, M. Chwalla, M. Hennrich, and R. Blatt, Phys. Rev. Lett. 110, 070403 (2013).

[30] C. Eltschka and J. Siewert, Sci. Rep. 2, 942 (2012).

[31] V. Coffman, J. Kundu, and W. K. Wootters, Phys. Rev. A 61, 052306 (2000).

[32] F. Verstraete, J. Dehaene, and B. DeMoor, Phys. Rev. A 68, 012103 (2003).

[33] C. Eltschka and J. Siewert, Phys. Rev. Lett. 108, 020502 (2012).

[34] W. Dür, G. Vidal, and J. I. Cirac, Phys. Rev. A 62, 062314 (2000).

[35] A. Acin, D. Bruß, M. Lewenstein, and A. Sanpera, Phys. Rev. Lett. 87, 040401 (2001).

[36] A. Uhlmann, Open Syst. Inf. Dyn. 5, 209 (1998).

[37] C. Eltschka and J. Siewert, Quant. Inf. Comput. 13, 210 (2013).

[38] K. G. H. Vollbrecht and R. F. Werner, Phys. Rev. A 64, 062307 (2001).

[39] C. H. Bennett, D. P. DiVincenzo, J. A. Smolin, and W. K. Wootters, Phys. Rev. A 54, 3824 (1996).

[40] J. M. Leinaas, J. Myrheim, and E. Ovrum, Phys. Rev. A 74, 012313 (2006).

[41] M. G. A. Paris and J. Rehacek (eds.), Quantum State Estimation, Lecture Notes in Physics, Vol. 649 (Springer, Heidelberg, 2004).

[42] O. Gühne, C.-Y. Lu, W.-B. Gao, and J.-W. Pan, Phys. Rev. A 76, 030305 (2007).

[43] G. Tóth, W. Wieczorek, D. Gross, R. Krischek, C. Schwemmer, and H. Weinfurter, Phys. Rev. Lett. 105, 250403 (2010).

[44] C. Eltschka, A. Osterloh, and J. Siewert, Phys. Rev. A 80, 032313 (2009). 